\title{
ModPhred: an integrative toolkit for the analysis and storage of nanopore sequencing DNA and RNA modification data
}

\author{
Leszek P. Pryszcz $(\mathbb{1})^{1,2, *}$ and Eva Maria Novoa $\mathbb{1}^{1,3, *}$
}

${ }^{1}$ Centre for Genomic Regulation (CRG), The Barcelona Institute of Science and Technology, Barcelona 08003, Spain, ${ }^{2}$ International Institute of Molecular and Cell Biology, 02-109 Warsaw, Poland and ${ }^{3}$ Universitat Pompeu Fabra (UPF), Barcelona, Spain

*To whom correspondence should be addressed.

Associate Editor: Peter Robinson

Received on March 26, 2021; revised on July 7, 2021; editorial decision on July 15, 2021; accepted on July 21, 2021

\begin{abstract}
Motivation: DNA and RNA modifications can now be identified using nanopore sequencing. However, we currently lack a flexible software to efficiently encode, store, analyze and visualize DNA and RNA modification data.

Results: Here, we present ModPhred, a versatile toolkit that facilitates DNA and RNA modification analysis from nanopore sequencing reads in a user-friendly manner. ModPhred integrates probabilistic DNA and RNA modification information within the FASTQ and BAM file formats, can be used to encode multiple types of modifications simultaneously, and its output can be easily coupled to genomic track viewers, facilitating the visualization and analysis of DNA and RNA modification information in individual reads in a simple and computationally efficient manner.

Availability and implementation: ModPhred is available at https://github.com/novoalab/modPhred, is implemented in Python3, and is released under an MIT license. Docker images with all dependencies preinstalled are also provided.

Contact: Ipryszcz@crg.eu or eva.novoa@crg.eu

Supplementary information: Supplementary data are available at Bioinformatics online.
\end{abstract}

\section{Introduction}

Third generation sequencing technologies have revolutionized our ability to identify base modifications in single molecules (Garalde et al., 2018; Kelleher et al., 2018; Liu et al., 2019b; Loman et al., 2015; Novoa et al., 2017). While many tools have been developed in the recent years to detect DNA and RNA modifications from nanopore sequencing datasets (Begik et al., 2021; Jenjaroenpun et al., 2021; Leger et al., 2019; Liu et al., 2019a,c; Ni et al., 2019; Pratanwanich et al., 2020; Stoiber et al., 2017; Yuen et al., 2020), there are limited tools allowing retrieval, storage, manipulation and visualization of modification information (De Coster et al., 2020; Leger, 2020).

Currently, the only available algorithm to extract and store DNA or RNA modification information from basecalled FAST5 datasets is megalodon (https://github.com/nanoporetech/megalo don), a tool developed by Oxford Nanopore Technologies (ONT) that relies on a previously trained basecalling model to extract methylation information from each raw Fast5 read, which is then dumped into a plain text file that will contain all predicted modified sites. However, megalodon presents several caveats and limitations: (i) it only supports $\mathrm{m}^{5} \mathrm{C}$ and $\mathrm{m}^{6} \mathrm{~A}$ DNA modification detection, (ii) it cannot be used with direct RNA sequencing datasets that are mapped to the genome, (iii) it does not integrate modification information within the FastQ format, (iv) it does not have the ability to encode multiple RNA modification types simultaneously (e.g. $\mathrm{m}^{5} \mathrm{C}$ and $\mathrm{hm}^{5} \mathrm{C}$ ), (v) it cannot be parallelized by splitting the input FAST5 files into separate read chunks and (vi) it does not offer options for downstream analyses or visualization of the results (Supplementary Table S1).

Here, we present ModPhred, a toolkit that encodes DNA and/or RNA modification information within the FastQ and BAM formats, allowing its analysis and visualization at single molecule resolution (Fig. 1A). We show that ModPhred can extract and encode modification information from basecalled FAST5 datasets 4-8 times faster than megalodon, while producing output files that are 20-50 times smaller (Supplementary Table S2). Finally, we illustrate the applicability of the ModPhred toolkit for the analysis of both DNA and RNA modifications. The toolkit is easy to use by the non-bioinformatic expert, and generates user-friendly reports to facilitate the downstream analyses as well as several forms of visualization of the modification information (Fig. 1B), both at per-site as well as at per-read level.

\section{Materials and methods}

ModPhred is conceived to efficiently encode, process and visualize DNA and RNA modification data from nanopore sequencing 
A

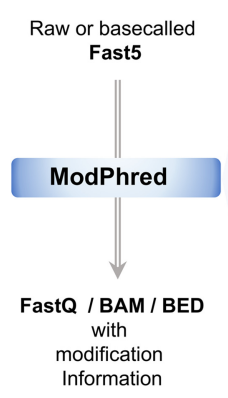

modification
1. ENCODE MODIFICATIONS into FASTQ files

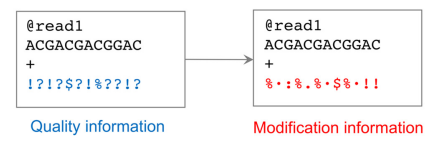

2. EXTRACT PER-POSITION MODIFICATION SCORES

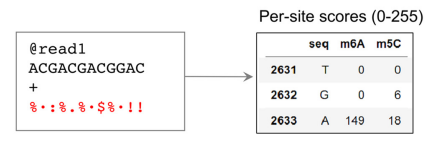

3. VISUALIZE MODIFICATIONS and CO-OCCURRENCE Genomic tracks

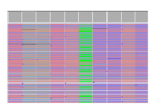

$m^{6} \mathrm{~A}$

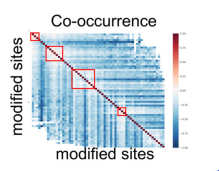

D
E. coli K12 genome
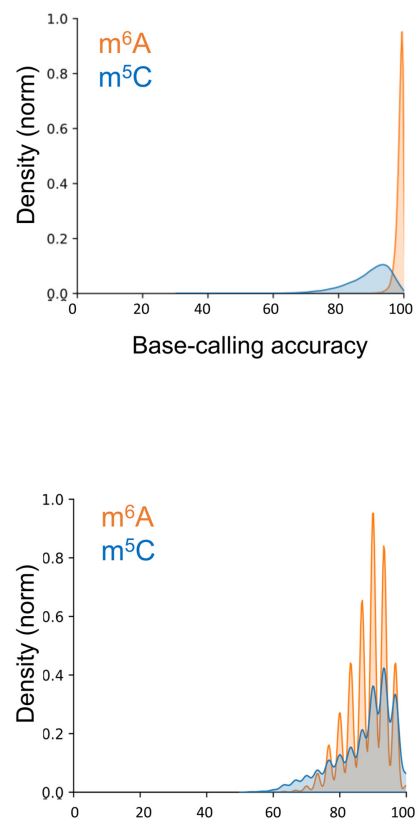

Median modification probability
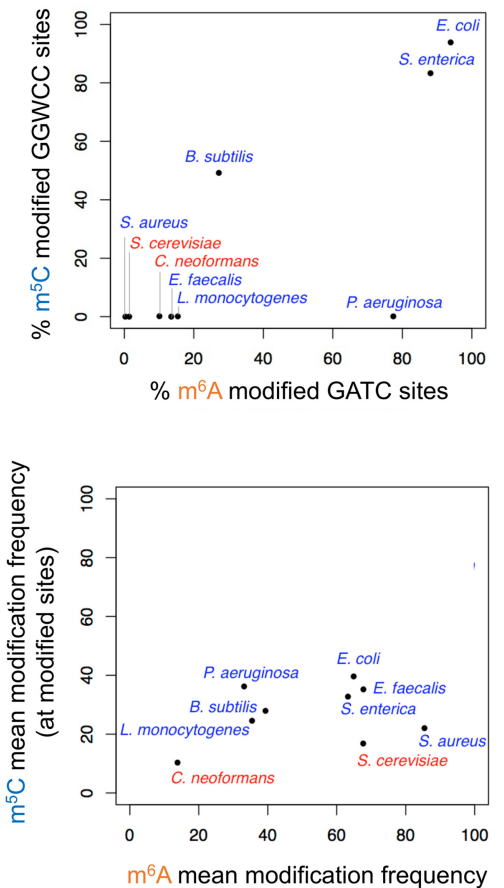

(at modified sites)
B
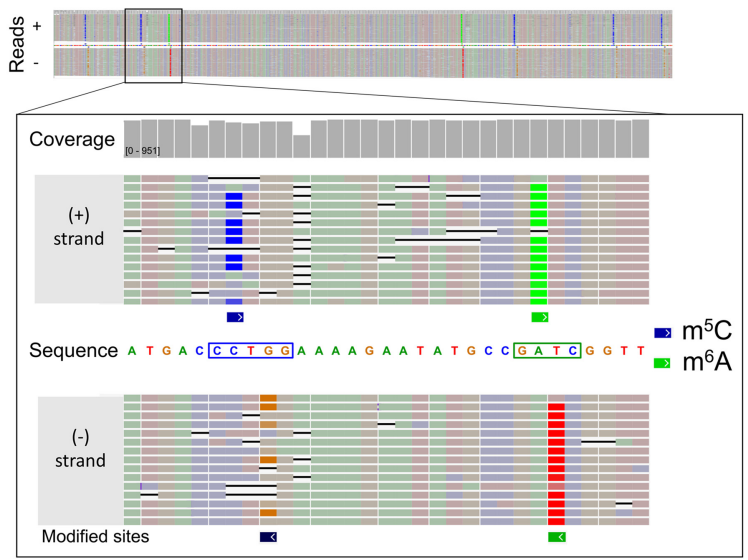

ZymoBIOMICS microbial genome standards

$m^{6} A$ (NC_000913.3:1-25000)

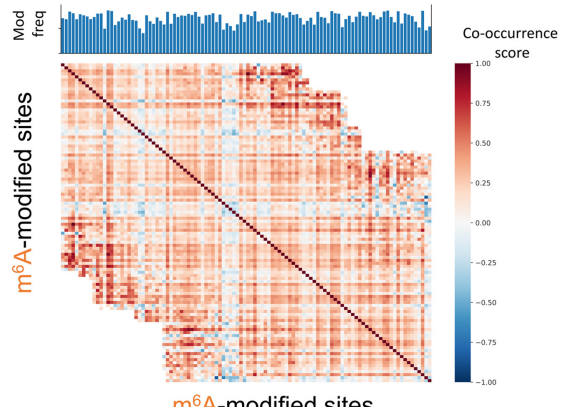

$m^{5} \mathrm{C}$ (NC_000913.3:1-25000)

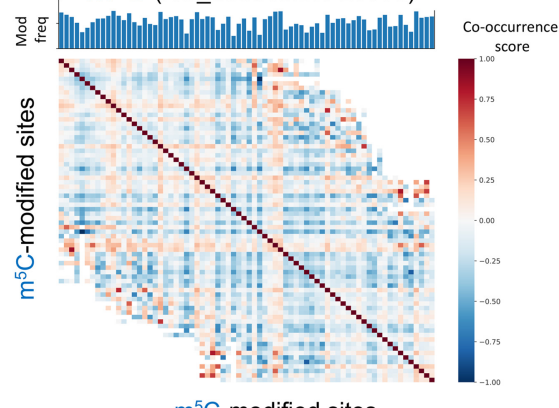

$\mathrm{m}^{5} \mathrm{C}$-modified sites

Fig. 1. Overview of ModPhred. (A) Schematic representation of ModPhred input, output and steps performed. Briefly, ModPhred uses as input raw or basecalled Fast5, and returns FASTQ, BAM and BEDGraph with modification information. To achieve this, ModPhred first encodes modification information into FASTQ files (modEncode) substituting the quality information, and then into the BAM files (modAlign). ModPhred can then easily extract modification information from BAM files to generate reports (modReport). Finally, modPhred can be used to visualize the results (modAnalysis). See also Supplementary Figure S1 for additional details on each of the four individual modules of ModPhred. (B) IGV visualization of BAM files generated using ModPhred. Since ModPhred stores modification information in the base quality field, per-read modification information can be visualized in IGV browser by coloring reads based on per-base quality information. (C) Density plots of basecalling accuracies (upper panel) and median modification probabilities (lower panel) at predicted modified sites, generated by modReport. See also Supplementary Figure S2. (D) Analysis of ZymoBIOMICS microbial reference using ModPhred. In the left panels, global analysis of $\mathrm{m}^{6} \mathrm{~A}$ and $\mathrm{m}^{5} \mathrm{C}$ modification levels across different species are shown. In the right panels, co-occurrence analysis of $\mathrm{m}^{6} \mathrm{~A}$ (upper panel) and $\mathrm{m}^{5} \mathrm{C}$ (lower panel) DNA modifications are depicted, for the same genomic region (NC_000913.3_1-25000)

datasets. ModPhred only requires as input a reference genome and reads in FAST5 format, which can be raw (non-basecalled) or basecalled using RNA or DNA modification-aware basecaller (guppy version $\geq 3.4)$. ModPhred extracts and integrates DNA/RNA modification information into the FASTQ and BAM files, by including: (i) information regarding the type of DNA/RNA modification (e.g. $\mathrm{m}^{5} \mathrm{C}$ or $\mathrm{hm}^{5} \mathrm{C}$ ), and (ii) probability scores of the most probable modification, for each nucleotide in each read. By decoupling the processes of basecalling and modification annotation, ModPhred can rapidly extract the list of modifications without the need of recomputing the basecalling step.

ModPhred is subdivided into 4 modules, and performs the following tasks: (i) encoding of modification probabilities in FastQ, with optional basecalling step (modEncode); (ii) alignment of reads that includes generation of BAM files with modification information (modAlign); (iii) extraction of modification information from mapped reads (modReport); (iv) downstream analyses (modAnalysis), which include plotting of DNA/RNA modifications 
within genomic track viewers (modPlot), computing correlations between modified positions (modCorrelation) and read clustering based on modification patterns (modCluster) (Supplementary Fig. S1, see also Supplementary Methods).

Firstly, ModEncode processes the FAST5 reads and stores the most likely type of modification for each base in every read. This is achieved by encoding the modification probability in the form of an ASCII character, replacing the basecalling qualities that are by default encoded in FastQ files (Supplementary Fig. S1, see also Supplementary Note S1). Such storage of information results in data compression, simplicity and versatility. Since probability of modification is stored inside the FastQ file, no external databases or additional files are needed for calculation or visualization of modifications. Per-base modification probabilities are also stored in BAM files that are derived from FastQ during the alignment step, which is performed by modAlign. modReport then calculates a list of statistics for every base of the genome and reports positions that are modified. Finally, modAnalysis generates graphical representations of modification statistics, as well as high-level analysis of DNA/RNA modification distributions, including co-occurrence of modifications and per-read clustering based on similarity of DNA/ RNA modification patterns.

\section{Implementation of ModPhred}

We first tested ModPhred for the annotation and analysis of DNA modifications in microbial datasets (Supplementary Table S3). We should note that current guppy basecalling models (versions 3.2.1 and later) can so far only detect $\mathrm{m}^{5} \mathrm{C}$ in $\mathrm{CCWGG}$ and $\mathrm{CpG}$ contexts, and $\mathrm{m}^{6} \mathrm{~A}$ in GATC contexts. Therefore, our analysis was limited to these modifications and sequence contexts. To this end, we analyzed a high-coverage $(900 \times)$ E.coli K12 DNA genome sequencing dataset. ModPhred reported $38897 \mathrm{~m}^{6} \mathrm{~A}$ and 29 $165 \mathrm{~m}^{5} \mathrm{C}$-modified positions in the E.coli chromosome, with mean modification frequencies at the modified sites of $63.2 \%$ and $42.4 \%$ (Supplementary Fig. S2A and B) and mean modification probabilities of 0.878 and 0.865 for $\mathrm{m}^{6} \mathrm{~A}$ and $\mathrm{m}^{5} \mathrm{C}$, respectively (Fig. 1C, see also Supplementary Table S4). ModPhred reported 99.7\% of E.coli GATC and CCWGG sites as 'modified' (i.e. modification frequency was greater than 0.05 ), whereas only $0.12 \%$ of $\mathrm{CpG}$ sites were reported as modified. The latter are expected to be false positives, since $\mathrm{CpG}$ methylation is not known to exist in E.coli. Similar results were observed in a second E.coli dataset with lower coverage $(250 \times)$, showing high reproducibility across datasets (Supplementary Fig. S2A and B, see also Supplementary Table S4).

We then applied ModPhred to the ZymoBIOMICS microbial DNA reference dataset (Supplementary Table S3). We find that $\mathrm{m}^{6} \mathrm{~A}$ and $\mathrm{m}^{5} \mathrm{C}$ predictions, both in terms of modification frequency as well as in terms of penetrance, largely vary across species. E.coli showed the highest penetrance of $\mathrm{m}^{6} \mathrm{~A}$ and $\mathrm{m} 5 \mathrm{C}$ modifications in GATC and CCWGG sequence contexts, in agreement with previous results (Fig. 1D). Closely related species, such as S.enterica, showed similar penetrance of $\mathrm{m} 6 \mathrm{~A}$ and $\mathrm{m} 5 \mathrm{C}$ modifications in GATC and CCWGG sequence contexts. However, the vast majority of species analyzed did not show high penetrance of $\mathrm{m}^{5} \mathrm{C}$ modifications in CCWGG sites, suggesting that either the penetrance in these species is either low, or that the motif in which $\mathrm{m}^{5} \mathrm{C}$ is embedded is different than CCWGG (Fig. 1D, see also Supplementary Table S4).

Finally, we applied modPhred to direct RNA nanopore sequencing datasets. However, we should note that currently, there are no publicly available guppy models for the detection of RNA modifications. Thus, to illustrate the applicability of modPhred in direct RNA sequencing data, we employed an in-house taiyakitrained RNA modification-aware model that was trained using synthetic RNA molecules (we should note that this model isn't applicable to biological samples, see Supplementary Methods). Specifically, we examined the ability of modPhred to predict and annotate RNA modifications in different mixes of RNA-modified datasets, finding that modPhred accurately recapitulates the expected RNA modification frequencies (Supplementary Table S5, see also Supplementary Fig. S2C). Moreover, we illustrate how modPhred can be used for per-read cluster analysis based on their RNA-modification profiles, illustrating its applicability to identify read populations with similar co-occurrence of RNA modification patterns (Supplementary Fig. S2D). Overall, our results show that modPhred can be applied both for the analysis of DNA and RNA modifications in genomic and transcriptomic datasets.

\subsection{Benchmarking of modPhred and comparison to available tools}

ModPhred runtimes were compared to megalodon on two publicly available genome sequencing datasets: (i) the E.coli DNA genome sequencing (PRJEB22772) and (ii) the ZymoBIOMICS microbial reference DNA genome sequencing (PRJNA477598) datasets (Supplementary Table S2, see also Supplementary Fig. S3). We observed that ModPhred was $4-8 \times$ faster than megalodon, while producing $20-50 \times$ smaller result files than megalodon. Moreover, we found that megalodon was poorly applicable to high coverage samples (PRJEB22772, E.coli sample with $900 \times$ coverage) as the process would not finish after $120 \mathrm{~h}$, limiting megalodon's applicability in high coverage and/or large genomes (Supplementary Table S2). By contrast, we observed that the runtime of modPhred scaled well both with coverage and genome size, and that it was mainly limited by the speed of basecalling process (Supplementary Tables S6-S8). This limitation can be easily overcome by using a multi-GPU system as well as by processing each project or sample in smaller batches. Finally, we should note that modPhred can perform remote basecalling, allowing many remote clients to process read batches in parallel from multiple workstations (or computing cluster nodes). By contrast, megalodon is designed to process all reads from a given sample at once and in a single workstation equipped with one or more dedicated GPUs, which leads to decreased parallelization and increased computing times (Supplementary Table S3, see also Supplementary Note S2).

\section{Acknowledgement}

The authors thank the CRG Scientific Information Technologies (SIT) facility for setting up the GPU cluster and continuously upgrading the corresponding software that was needed to benchmark modPhred and megalodon with diverse guppy versions.

\section{Funding}

L.P.P. was supported by funding from the European Union's H2020 research and innovation programme under Marie Sklodowska-Curie grant agreement No. 754422. This work was partly supported by the Spanish Ministry of Economy, Industry and Competitiveness (MEIC) [PGC2018-098152-A-100 to E.M.N.]. They acknowledge the support of the Spanish Ministry of Science and Innovation to the EMBL partnership, Centro de Excelencia Severo Ochoa and CERCA Programme/Generalitat de Catalunya.

\section{Conflict of Interest}

EMN has received travel and accommodation expenses to speak at Oxford Nanopore Technologies conferences. All authors declare that the research was conducted in the absence of any commercial or financial relationships that could be construed as a potential conflict of interest.

\section{Data availability}

Nanopore DNA sequencing raw Fast 5 were obtained from publicly available datasets (E. coli: PRJEB22772; Zymo mock community: PRJNA477598) from previously published studies (McIntyre et al., 2019; Jain et al., 2017). Direct RNA sequencing datasets with and without RNA modifications were 
obtained from publicly available datasets (m6A: PRJNA511582; m5C: PRJNA563591; hm5C: PRJNA548268; UNM:PRJNA511582) (Liu et al., 2019a; Begik et al., 2021).

\section{References}

Begik,O. et al. (2021) Quantitative profiling of pseudouridylation dynamics in native RNAs with nanopore sequencing. Nat. Biotechnol., doi: 10.1038/s41587-021-00915-6.

De Coster,W. et al. (2020) Methplotlib: analysis of modified nucleotides from nanopore sequencing. Bioinformatics, 36, 3236-3238.

Garalde,D.R. et al. (2018) Highly parallel direct RNA sequencing on an array of nanopores. Nat. Methods, 15, 201-206.

Jain,M. et al. (2017) MinION Analysis and Reference Consortium: Phase 2 data release and analysis of R9.0 chemistry. F1000Res., 6, 760.

Jenjaroenpun,P. et al. (2021) Decoding the epitranscriptional landscape from native RNA sequences. Nucleic Acids Res., 49, e7.

Kelleher,P. et al. (2018) Identification of DNA base modifications by means of pacific biosciences RS sequencing technology. Methods Mol. Biol., 1681, $127-137$.

Leger,A. (2020) a-slide/pycoMeth: v0.4.25.

Leger,A. et al. (2019) RNA modifications detection by comparative Nanopore direct RNA sequencing. BioRxiv, doi: 10.1101/843136.
Liu,H. et al. (2019a) Accurate detection of m6A RNA modifications in native RNA sequences. Nat. Commun., 10, 4079.

Liu,Q. et al. (2019b) Detection of DNA base modifications by deep recurrent neural network on Oxford Nanopore sequencing data. Nat. Commun., 10, 2449.

Liu, Q. et al. (2019c) NanoMod: a computational tool to detect DNA modifications using Nanopore long-read sequencing data. BMC Genomics, 20,78 .

Loman,N.J. et al. (2015) A complete bacterial genome assembled de novo using only nanopore sequencing data. Nat. Methods, 12, 733-735.

McIntyre,A.B.R. et al. (2019) Single-molecule sequencing detection of N6-methyladenine in microbial reference materials. Nat. Commun., 10, 579.

Ni,P. et al. (2019) DeepSignal: detecting DNA methylation state from Nanopore sequencing reads using deep-learning. Bioinformatics, 35, 4586-4595.

Novoa,E.M. et al. (2017) Charting the unknown epitranscriptome. Nat. Rev. Mol. Cell Biol., 18, 339-340.

Pratanwanich,P.N. et al. (2020) Detection of differential RNA modifications from direct RNA sequencing of human cell lines. bioRxiv, doi: 10.1101/2020.06.18.160010

Stoiber,M. et al. (2017) De novo identification of DNA modifications enabled by genome-guided nanopore signal processing. bioRxiv, doi: 10.1101/094672.

Yuen,Z.W.-S. et al. (2020) Systematic benchmarking of tools for $C p G$ methylation detection from nanopore sequencing. bioRxiv, doi: 10.1101/2020.10.14.340315. 